\title{
The Effect of Collagen-Chitosan-Sodium Hyaluronate Implantation Composite on Inflammation Reaction (Flare) In New Zealand Rabbit Corneal Stroma
}

\author{
Diana Akmalin, Reni Prastyani*, Prihartini Widiyanti \\ Clinical Medicine Faculty of Medicine Universitas Airlangga Surabaya Indonesia 60286 \\ *Corresponding author details: Reni Prastyani; renipraunair@gmail.com
}

\begin{abstract}
Corneal transplantation is a solution in treating visual disturbances due to corneal opacities, but this procedure is said to be limited. Various efforts have been made in the development of alternative therapies, one of which is by using composite biomaterials. For this reason, this study aimed to analyze the effect of the collagen-chitosansodium hyaluronate composite on the inflammatory reaction (flare) in the corneal stromal injury of New Zealand rabbits. The method used was thirty adult male New Zealand rabbits which were divided into three groups. The first group (negative control) was not treated, the second group (positive control) was subjected to intrastromal injuries by making a stromal pocket with the help of a crescent knife, and the third group had a collagen-chitosansodium hyaluronate biomaterial implanted in the stromal pocket. The results of this study showed that there were significant differences in inflammatory reactions (flares) in the collagen-chitosan-sodium hyaluronate composite implantation group with the control and positive control groups ( $p=0.001,>0.01)$. Therefore, it can be concluded that the inflammatory reaction (flare) in the implanted group was higher than the control group, this could occur due to the short observation period so that the biomaterial was not completely absorbed.
\end{abstract}

Keywords: inflammatory reactions (flares); biomaterials; collagen; chitosan; sodium hyaluronate

\begin{abstract}
INTRODUCTION
Corneal disease is the fifth leading cause of blindness in the world, following cataracts, refractive errors, glaucoma, and age-related macular degeneration. A total of 216.6 million people in the world are recorded as having visual impairments with 4.5 million of them having problems due to loss of corneal clarity and most of them occur in the population at a young age. Corneal transplantation is a solution in treating visual disturbances due to corneal opacities, but this procedure is limited. This is due to the shortage of tissue donors and the risk of immunological rejection after the graft is placed, which is a high cost and time-consuming condition in the rehabilitation process. (Mathews et al., 2018).
\end{abstract}

The cornea is the most important part of the eye and has two main functions. First, the epithelial layer of the cornea of the eye serves as a physical barrier that prevents harmful agents from entering the eye. Second, it plays an active role in the process of vision by contributing $70 \%$ of its refractive power. The cornea is transparent with a curvature that allows light to be focused and transmitted through the lens to the retina. Therefore, the integrity of the corneal tissue is very important to produce good vision (Grolik et al., 2015) (Bukowiecki et al., 2017) (Mathews et al., 2018). Various efforts have been made in the development of alternative therapies for corneal blindness focused on tissue engineering. This strategy involves the use of cells, biomolecules (growth factors) that promote cell proliferation and ensure proper expression of biomarkers, as well as the biomaterials to be used (Bukowiecki et al., 2017) (Mathews et al., 2018) (Amouzegar et al., 2016) (Jorge E et al., 2016).

Visual disturbances are initiated when there is direct injury to the stroma or there is an unintacted epithelial layer.
The collagen-chitosan-sodium hyaluronate biomaterial has also been tested in vitro as an artificial cornea which shows that there is no chemical interaction between the biomaterials that can support cellular interactions and provide hydration to maintain eye clarity and permeability, morphologically similar to keratocytes so that it is expected to help the corneal healing process and avoid clouding of the corneal stroma (Widiyanti \& Prastyani, 2019) (X. Li et al., 2013). Through this description, this study was prepared with the aim of analyzing the effect of the collagen-chitosan-sodium hyaluronate composite on the inflammatory reaction (flare) in the corneal stromal injury of New Zealand rabbits.

\section{METHOD}

This study is a true experimental with an in vivo randomized control group post test only design. This study examines the corneas obtained from the eyeballs of New Zealand rabbits which were treated with an implant of the collagen-chitosan-sodium hyaluronate composite biomaterial with different inflammatory reactions (flares) in the corneal tissue of New Zealand rabbits. The test was carried out on three groups, namely without injury, cornea with injury to the stroma, and with and without biomaterial implants. This experimental study analyzed three groups. One observation was made in each group, namely after 2 weeks from the start of the study.

Furthermore, the population of this study was the cornea of adult New Zealand white rabbits aged 12-18 months with a bodyweight of $3.0-3.5 \mathrm{~kg}$. Meanwhile, the research data were collected in a special data collection sheet and then grouped, presented in tabulated form, and analyzed descriptively. 
Then, the comparison of variables between groups on a ratio scale was analyzed using the Kruskal-Wallis test. On the variables with an interval, scales were analyzed using the normality test, then continued with Annova if the distribution was normal or Kruskal-Wallis if the distribution was not normal.

\section{RESULT}

\section{Characteristics of Research Sample}

This research was conducted at the Faculty of Veterinary Medicine, Universitas Airlangga using the cornea of a male New Zealand white rabbit (Oryctolagus cuniculus) aged 12-18 months and weighing 3.0-3.5 kg. During the research, rabbits received the same treatment, food was given in the form of pallets and drinks in the form of mineral water that had been adjusted to the same portion. This study was conducted on 30 rabbit eye corneas which were divided into three groups. The first group was a negative control group (K-) where 10 rabbit corneas were not treated. The second group was a positive control group $(\mathrm{K}+)$, namely a group of 10 rabbit eye corneas that received intrastromal corneal injury treatment using crescents. Meanwhile, the third group was the Treatment Group (KP) wherein this group ten rabbit eyes were injured in the form of a stromal pouch using a crescent intrastromal, which was then implanted with a collagen-chitosansodium hyaluronate composite biomaterial.

In rabbits with the treatment group on the cornea, stromal pouches were made with a thickness of $0.2-0.3 \mathrm{~mm}$ and a diameter of $4 \mathrm{~mm}$. Then the collagen-chitosan-sodium hyaluronate composite biomaterial with a diameter of 3 $\mathrm{mm}$ and a thickness of $0.2 \mathrm{~mm}$ was inserted into the stromal sac.

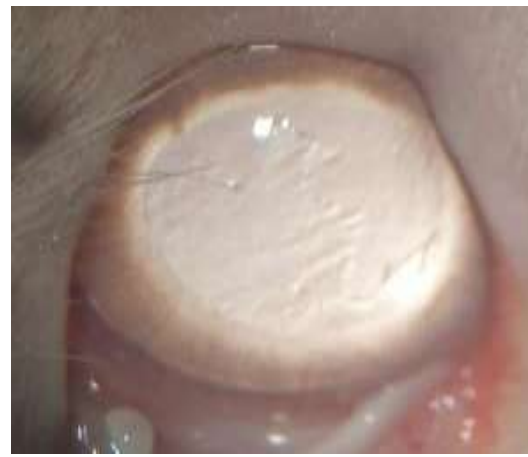

Control (-)
In the treatment group, post-implantation composite suturing was not performed and no biomaterial extrusion was noted during the observation until the 14th day. On the 14th day of observation using a handheld slit lamp, there was no intrastromal biomaterial degradation in all observed eyes, but there were no signs of eye infection or severe rejection during 14 days of observation in all rabbits with collagen-chitosan-sodium composite implants. intrastromal hyaluronic acid.

\section{Inflammatory reactions (flares)}

The inflammatory reaction in the anterior chamber of the eye is in the form of a flare which is a plasma protein in the anterior chamber of the eye so that it comes out of the blood vessels due to injury to the cornea. Examination of the inflammatory reaction was carried out on the 14th day after treatment, using a handheld slit lamp biomicroscopy. The results of the examination are then classified as 0 if there is no visible flare, 1 if there is a little flare, 2 Moderate (visible flare, visible iris and lens detail), 3 Marked (clearly visible flare, blurred iris, and lens detail), 4 Intense (exuded exudate, iris, and the lens are not visible).

It is shown that Figure 1 is a comparison of the inflammatory reactions in the three groups observed. In the positive control group and the negative control group, there was no flare, while in the treatment group 6 out of 10 rabbit eyes had an inflammatory reaction in the anterior chamber, namely flare around the biomaterial. There were four eyes with slight flare ( +1 value) and one eye (image $1 d$ ) with visible flare but visible iris and lens detail $(+2)$.

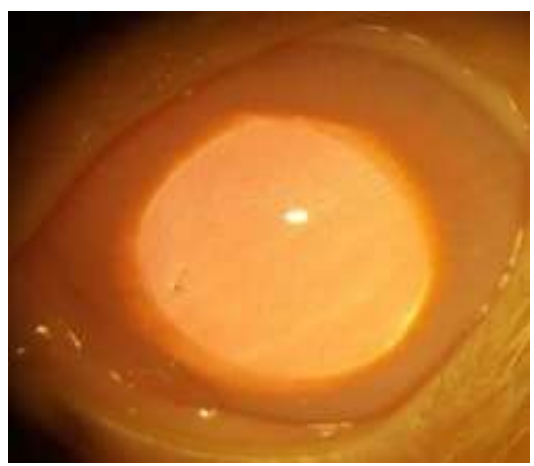

Control $(+)$

Treatment
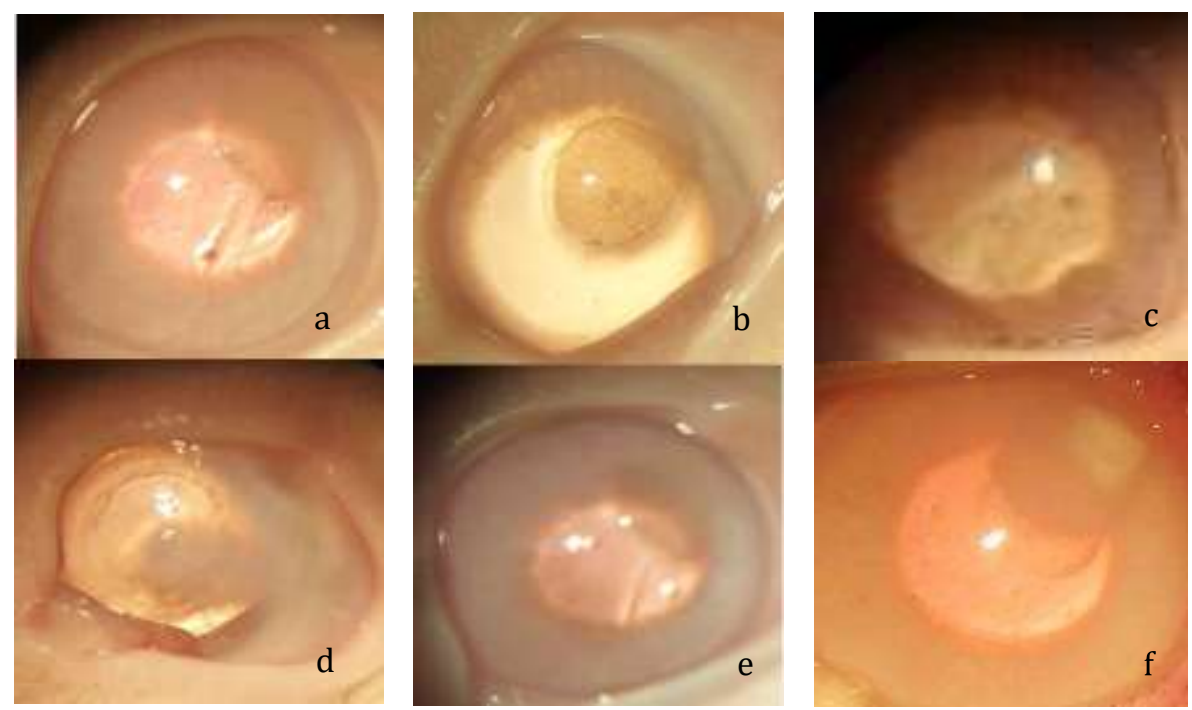

FIGURE 1: Inflammatory reaction (flare) observed based on iris and lens details 
Clinical examination of inflammatory reactions in the anterior chamber in the form of flares was carried out in thirty rabbit eyes. Table 1 shows that the results of statistical analysis showed significant differences between the negative control group, positive control group, and the stromal treatment group with the implantation of the collagen-chitosan-sodium hyaluronate biomaterial composite $(\alpha<0.01)$.

TABLE 1: Flare distribution

\begin{tabular}{ccccccc}
\hline \multirow{2}{*}{ Group } & \multicolumn{7}{c}{ Flare Expression } \\
\cline { 2 - 6 } & $\mathbf{n}$ & Mean & STD & Min. & Max. & p-Value \\
\hline K- & 10 & 0 & 0 & 0 & 0 & $0,001^{* *}$ \\
K+ & 10 & 0 & 0 & 0 & 0 & \\
KP & 10 & 0,7 & 0,67 & 0 & 2 & \\
\hline
\end{tabular}

Note: STD: standard deviation; Min.: minimal; Max.:max; $\left.{ }^{* *}\right)$ significant $<0.01$ using the kruskall wallis test

\section{DISCUSSION}

The cornea is an avascular transparent tissue that functions as a physical barrier to the eye to prevent harmful agents from entering the eye and plays an active role in the visual process by contributing $70 \%$ of its refractive power. The five layers of the cornea have different regenerative abilities, with the stromal layer making up $80-85 \%$ of the cornea and providing many of the cornea's biochemical properties including refractive power, stability, and transparency. In conditions of inflammation of the cornea, corneal cells play an active role in synthesizing various proteins derived from plasma and certain cytokines.

Inflammatory cells can enter the cornea through defects that form on its surface and sometimes enter the cornea via interlamellar infiltration. Corneal damage to the stromal layer can be caused by corneal surgery, refractive surgery, trauma, or infection will cause corneal opacities that occur due to the extracellular matrix and imperfect collagen fiber arrangement. Turbidity in the stroma will reduce the clarity of the cornea so that its refractive function will decrease and result in blindness (Antaki et al., 2020) (Mathews et al., 2018) (Doctor \& Working, 2018) (Shenoy et al., 2009).

Currently, tissue engineering techniques and regenerative medicine are known to continue to develop in creating biomaterials that have properties and characteristics that match natural tissues. Biomaterials form tissues and organs in the human body so that several components are needed to be able to meet the characteristics that are in accordance with physiological conditions without causing side effects. Corneal tissue engineering requires good biocompatibility, which has a three-dimensional structure, has characteristics like the human cornea, and the level of degradation that can be adjusted to the rate of degradation of tissues and organs formed by implanted corneal cells.

Biomaterials used today are made of synthetic and natural polymers. Synthetic polymers are more biocompatible and do not stimulate excessive immunological reactions, and can be mixed with other materials to achieve the desired goal. Various kinds of corneal biomaterials have been developed in the last five years as an effort to help the healing of the cornea including amnion membrane, chitosan, collagen, silk fiber, gelatin, and sodium hyaluronate. A good biomaterial composition can reduce the accumulation and turnover of extracellular matrix components in tissue injury, mediate immune responses, prevent neovascularization and reduce fibroblast deposition thereby reducing scar tissue formation (Ke \& Wang, 2017) (Ueno et al., 2005).

The extracellular matrix of the corneal stroma mainly consists of type I collagen with a lower amount of type IV collagen and proteoglycans, in addition to the collagen network there are 3-5\% of keratocyte cells that make up the extracellular matrix and maintain the layer and clarity of the stroma (Knupp et al., 2009) (Hassell \& Birk, 2010) (Palchesko et al., 2018). Therefore, collagen biomaterials such as collagen gel, and acellular porcine cornea are widely used as potential corneal substitutes. Collagen is known to be a major component of the corneal stroma and is biochemically compatible with all corneal cells in vivo (Tanaka et al., 2011) (Xiao et al., 2011) (Yoeruek et al., 2012) (Jorge E et al., 2016).

It is now known that crosslinking collagen has been widely applied with the aim of increasing its mechanical strength and chemical stability without compromising biological advantages such as the addition of soluble tropocollagen to increase the transparency and strength of the material. In a study conducted by Grolik et. al., 2014 said that the collagen-chitosan membrane observed good physicochemical and biomechanical properties which were equivalent to the amnion membrane as a substitute for cell carriers in the wound healing process on the surface of the cornea of the eye.

Chitosan is a natural polymer material that is formed from the distillation of chitin and can form stable covalent bonds with other compounds so that it can support biomaterials to be implanted because it has non-toxic, biocompatible, and biodegradable properties. Howling et. al. said that in chitosan collagen composites, chitosan can form a thin membrane between collagen fibers which causes tension in the fibers to increase, besides that chitosan can also inhibit collagenase so that the composite is not degraded too quickly and improves the mechanical properties of the biomaterial. The chitosan collagen membrane composite showed better mechanical and optical strength properties when transplanted into porcine corneas, and allowed epithelial, stromal, and nerve regeneration within 12 months (Parekh et al., 2021).

During its development, sodium hyaluronic acid was added to chitosan collagen to form a collagen-chitosansodium hyaluronic complex. Sodium hyaluronate is one of several components, which is a natural glycosaminoglycan found in various connective tissues and can interact with water in the body so that it can expand the extracellular matrix. Sodium hyaluronate solution has also been used as a protective agent for ocular tissues and is currently used in intraocular surgery to protect the endothelium and form structures during surgery.

The collagen-chitosan-sodium hyaluronate complex which was observed in vivo in rabbit corneal stroma for five months showed that the complex was transparent, relatively strong but elastic. 
In particular, chitosan is proving to be a promising biomaterial for corneal bioengineering applications with good light transmittance, biocompatible and mechanical behavior. The complex of $20 \%$ collagen, $10 \%$ chitosan, and $0.5 \%$ sodium hyaluronate exhibited mildly weak corneal edema and other responses and had good corneal biocompatibility (Zhong et al., 2016).

In a recent study of the collagen-chitosan-sodium hyaluronate biomaterial, each component has different degradation capabilities for each component. In vitro cytotoxicity test showed that the composite was non-toxic and had antibacterial activity. In addition, the collagenchitosan-sodium hyaluronate biomaterial was also tested in vitro as to indicate the absence of chemical interactions between biomaterials that can support cellular interactions and provide hydration to maintain eye clarity and permeability, and in the presence of collagen is the most abundant extracellular matrix component in the cornea. does not stimulate an excessive inflammatory response when implanted in the eye (Widiyanti \& Prastyani, 2019) (Widiyanti \& Prastyani, 2020).

In this study, observations were made on the corneas of adult New Zealand male rabbits weighing approximately 1.5-3 kg which had been declared healthy by a veterinarian and had no abnormalities in the eyes, especially the cornea. Observations were then carried out on 3 groups of experimental animals consisting of 10 rabbits, namely, the untreated group, the corneal stromal injury group, and the corneal stromal injury group with the implantation of the collagen-chitosan-sodium hyaluronic biomaterial. Implantation of biomaterials was carried out by making a stromal sac using a crescent knife with an incision diameter of $4 \mathrm{~mm}$ and a thickness of $0.2-0.3 \mathrm{~mm}$, then the composite with a size of $3 \mathrm{~mm}$ was inserted into the stromal sac.

The process of implantation is identical to the study that was conducted by Chen in 2005, but the stromal sac formed and the implanted biomaterial was larger than the treatment in this study. In addition, Valdesz et.al. and Takiyama et al. has a biomaterial implantation technique that is almost the same as a larger biomaterial size, but in both studies, nylon 10.0 suturing was performed on the incision wound to avoid extrusion of the biomaterial (Jorge E et al., 2016). In this study, with a $3 \mathrm{~mm}$ diameter biomaterial implanted in a $4 \mathrm{~mm}$ diameter stromal sac without sutures, no biomaterial extrusion occurred within 2 weeks.

\section{Inflammatory Reactions (flares)}

In corneal wounds, an inflammatory reaction and posttraumatic endothelial dysfunction can occur, often causing epithelial and stromal edema, as well as an inflammatory reaction in the anterior chamber of the eye. Swollen endothelial cells called pseudoguttae are the result of an inflammatory reaction that is seen on examination by reflection from slit lamp light.

On examination, cells and flares will be found in the anterior chamber of the eye. Cells are a collection of inflammatory cells located in the anterior chamber of the eye. If there are too many inflammatory cells, they will settle under the anterior chamber of the eye to form a hypopyon. Flare is a plasma protein in the anterior chamber of the eye as a result of the blood aqueous barrier, so that plasma proteins come out of the blood vessels (Ciledag et al., 2010).

Researchers evaluated the inflammatory reaction in the form of flares in the anterior chamber of the eye. On examination, data obtained from 6 of 10 eyes that received treatment with collagen-chitosan-sodium hyaluronate biomaterial implantation with positive flare results. Anterior chamber reaction in the anterior chamber is one of the earliest signs of rejection in patients after keratoplasty, but the inflammatory reaction returns to normal 6 weeks after the procedure in both humans and animals (Küchle et al., 1994).

Meanwhile, Jennifer's research in 2012 stated that there was rejection after Descemet's Stripping Automated Endothelial Keratoplasty (DSAEK) was performed. It is known that in this study the assessment of acute rejection episodes in this study was carried out by observing the findings of Keratic precipitates (KPs) with or without corneal edema or the presence of flares in the anterior chamber with or without post-graft corneal edema. In observations, it was found that forty-five eyes experienced rejection with the majority of clinical signs appearing at 12 to 18 months after the procedure, but five of the signs appeared less than 6 months of observation (J. Y. Li et al., 2012).

However, according to research conducted by Baydoun et.al. flares will rapidly decrease and stabilize after a one month keratoplasty operation and if the inflammatory reaction (flare) increases on observation for a longer time it can strengthen the suspicion of a rejection. This correlates with this study, where in the six eyes that received the treatment the biomaterial composite was not completely absorbed and the observations made in this study two weeks after the corneal stromal injury and biomaterial implantation.

It is known that signs of inflammation in the form of flares, cells to hypopyon (exudate) in the anterior chamber of the eye can occur due to trauma to the cornea which results in disruption of the blood aqueous barrier. In a study conducted by Jorge et. al. who observed the clinical condition after implantation of the collagen vitrigel membrane biomaterial which was compared with the chitosan membrane.

Furthermore, through observations made for one week, it was found that the acute inflammatory reaction was more severe in the form of exudate that filled the anterior chamber of the eye with chitosan membrane implantation, the condition of the anterior chamber when correlated with the gradation of flare in the anterior chamber at a value of +4 or intense. However, the inflammatory reaction was minimal in the cornea with the implantation of the vitrigel collagen biomaterial. According to Jorge et. al. in the study the presence of collagen as the most abundant component of the extracellular matrix in the cornea did not stimulate an excessive inflammatory response when implanted in the eye (Jorge E et al., 2016).

In four eyes that were treated with collagen-chitosansodium hyaluronate biomaterial implantation, there were no signs of inflammation (flare) in the anterior chamber where two of them found the biomaterial had been well absorbed on observations made for 2 weeks. This can indicate that the collagen-chitosan-sodium hyaluronate composite biomaterial can restore corneal clarity with minimal inflammation.

\section{CONCLUSION}

Therefore, it can be concluded that the implanted group of the collagen-chitosan-sodium hyaluronate composite biomaterial for two weeks had a higher inflammatory reaction compared to stromal injuries that were not given the composite biomaterial this could occur due to the short observation period so that the biomaterial was not completely absorbed. 


\section{REFERENCES}

[1] Amouzegar, A., Chauhan, S. K., \& Dana, R. (2016). Alloimmunity and tolerance in corneal transplantation. The Journal of Immunology, 196(10), 3983-3991.

[2] Antaki, F., Kahwati, G., Sebag, J., Coussa, R. G., Fanous, A., Duval, R., \& Sebag, M. (2020). Predictive modeling of proliferative vitreoretinopathy using automated machine learning by ophthalmologists without coding experience. Scientific Reports, 10(1), 1-10.

[3] Bukowiecki, A., Hos, D., Cursiefen, C., \& Eming, S. A. (2017). Wound-healing studies in cornea and skin: parallels, differences and opportunities. International Journal of Molecular Sciences, 18(6), 1257.

[4] Ciledag, A., Kaya, A., Erol, S., Sen, E., Celik, G., Cesur, S., Fidan, Y., \& Kinikli, S. (2010). The Comparison of Pleural Fluid TNF-\&\#945; and IL-10 Levels with ADA in Tuberculous Pleural Effusion. Current Medicinal Chemistry, 17(19), 2096-2100. https://doi.org/10.2174/092986710791233652

[5] Doctor, M., \& Working, S. (2018). ALLEGHENY COUNTY MEDICAL SOCIETY.

[6] Grolik, M., Szczubiałka, K., Wowra, B., Dobrowolski, D., Orzechowska-Wylęgała, B., Wylęgała, E., \& Nowakowska, M. (2015). Corneal epithelial scaffolds based on chitosan membranes containing collagen and keratin. International Journal of Polymeric Materials and Polymeric Biomaterials, 64(3), 140148.

[7] Hassell, J. R., \& Birk, D. E. (2010). The molecular basis of corneal transparency. Experimental Eye Research, 91(3), 326-335.

[8] Jorge E, V.-G., Guillermo, M., Judith, Z., Angel, Z.-P., Gabriela, B., Jorge A, C.-R., \& Jennifer, E. (2016). In Vivo Biocompatibility of Chitosan and Collagenvitrigel Membranes for Corneal Scaffolding: A Comparative Analysis. Current Tissue Engineering (Discontinued), 5(2), 123-129.

[9] Ke, F., \& Wang, Y. (2017). Clinical Application of Ophthalmic Implant Materials and Biocompatibility. MATEC Web of Conferences, 100, 4020.

[10] Knupp, C., Pinali, C., Lewis, P. N., Parfitt, G. J., Young, R. D., Meek, K. M., \& Quantock, A. J. (2009). The architecture of the cornea and structural basis of its transparency. Advances in Protein Chemistry and Structural Biology, 78, 25-49.

[11] Küchle, M., Nguyen, N. X., \& Naumann, G. O. H. (1994). Aqueous flare following penetrating keratoplasty and in corneal graft rejection. Archives of Ophthalmology, 112(3), 354-358.

[12] Li, J. Y., Terry, M. A., Goshe, J., Shamie, N., \& DavisBoozer, D. (2012). Graft rejection after Descemet's stripping automated endothelial keratoplasty: graft survival and endothelial cell loss. Ophthalmology, 119(1), 90-94.
[13] Li, X., Yang, Y., Li, Q., Dai, Y., Wang, C., \& Chen, J. (2013). Morphologic Characteristics and Proliferation of Rabbit Corneal Stromal Cells Onto Complexes of CollagenChitosan-Sodium Hyaluronate Under Simulated Microgravity. Investigative Ophthalmology \& Visual Science, 54(10), 6877-6885.

[14] Mathews, P. M., Lindsley, K., Aldave, A. J., \& Akpek, E. K. (2018). Etiology of global corneal blindness and current practices of corneal transplantation: a focused review. Cornea, 37(9), 1198-1203.

[15] Palchesko, R. N., Carrasquilla, S. D., \& Feinberg, A. W. (2018). Natural biomaterials for corneal tissue engineering, repair, and regeneration. Advanced Healthcare Materials, 7(16), 1701434.

[16] Parekh, M., Romano, V., Hassanin, K., Testa, V., Wongvisavavit, R., Ferrari, S., Haneef, A., Willoughby, C., Ponzin, D., \& Jhanji, V. (2021). Biomaterials for corneal endothelial cell culture and tissue engineering. Journal of Tissue Engineering, 12, 2041731421990536.

[17] Shenoy, R., Khandekar, R., Bialasiewicz, A. A., \& Muniri, A. Al. (2009). Corneal endothelium in patients with diabetes mellitus: a historical cohort study. European Journal of Ophthalmology, 19(3), 369-375.

[18] Tanaka, Y., Shi, D., Kubota, A., Takano, Y., Fuse, N., Yamato, M., Okano, T., \& Nishida, K. (2011). Irreversible optical clearing of rabbit dermis for autogenic corneal stroma transplantation. Biomaterials, 32(28), 6764-6772.

[19] Ueno, T., Omura, T., Takahashi, T., Matsumoto, H., Takahashi, Y., Kakita, A., \& Yamashina, S. (2005). Liver transplantation using liver grafts preserved under high pressure. Artificial Organs, 29(10), 849-855.

[20] Widiyanti, P., \& Prastyani, R. (2020). In Vitro Assay of Cornea Artificial Properties. Journal of International Dental \& Medical Research, 13(2).

[21] Widiyanti, P., \& Prastyani, R. (2019). CollagenChitosan-Glycerol-HPMC Composite as Cornea Artificial Candidate. Journal of Biomimetics, Biomaterials and Biomedical Engineering, 42, 14-21.

[22] Xiao, J., Duan, H., Liu, Z., Wu, Z., Lan, Y., Zhang, W., Li, C., Chen, F., Zhou, Q., \& Wang, X. (2011). Construction of the recellularized corneal stroma using porous acellular corneal scaffold. Biomaterials, 32(29), 6962-6971.

[23] Yoeruek, E., Bayyoud, T., Maurus, C., Hofmann, J., Spitzer, M. S., Bartz-Schmidt, K., \& Szurman, P. (2012). Reconstruction of corneal stroma with decellularized porcine xenografts in a rabbit model. Acta Ophthalmologica, 90(3), e206-e210.

[24] Zhong, J., Deng, Y., Tian, B., Wang, B., Sun, Y., Huang, H., Chen, L., Ling, S., \& Yuan, J. (2016). Hyaluronate acid-dependent protection and enhanced corneal wound healing against oxidative damage in corneal epithelial cells. Journal of Ophthalmology, 2016. 